\title{
WP 15-14
}

\author{
Nikola Gradojevic \\ IÉSEG School of Management (LEM-CNRS), Lille Catholic University, France \\ Faculty of Technical Sciences, University of Novi Sad, Serbia \\ The Rimini Centre for Economic Analysis, Italy
}

Marko Caric

Faculty of Economics and Engineering Management, Business Academy, Serbia

\section{MULTI-CRITERIA ClASSIFICATION FOR Pricing European OPTIONS}

Copyright belongs to the author. Small sections of the text, not exceeding three paragraphs, can be used provided proper acknowledgement is given.

The Rimini Centre for Economic Analysis (RCEA) was established in March 2007. RCEA is a private, nonprofit organization dedicated to independent research in Applied and Theoretical Economics and related fields. RCEA organizes seminars and workshops, sponsors a general interest journal The Review of Economic Analysis, and organizes a biennial conference: The Rimini Conference in Economics and Finance (RCEF) . The RCEA has a Canadian branch: The Rimini Centre for Economic Analysis in Canada (RCEACanada). Scientific work contributed by the RCEA Scholars is published in the RCEA Working Papers and Professional Report series.

The views expressed in this paper are those of the authors. No responsibility for them should be attributed to the Rimini Centre for Economic Analysis.

The Rimini Centre for Economic Analysis

Legal address: Via Angherà, 22 - Head office: Via Patara, 3 - 47921 Rimini (RN) - Italy

www.rcfea.org - secretary@rcfea.org 


\title{
Predicting Systemic Risk with Entropic Indicators
}

\author{
Nikola Gradojevic* $\quad$ Marko Caric $^{\dagger}$
}

2015

\begin{abstract}
This paper concentrates on quantifying the behavioral aspects of systemic risk by using a novel approach based on entropy. More specifically, we study aggregate market expectations and the predictability of the systemic risk before and during the financial crisis in 2008. Two underlying signals for estimating entropic risk measures are considered: 1) skewness premium of deepest out-of-the-money options, and 2) implied volatility ratio in regards to deepest out-of-the-money options. The findings confirm the predictive and contemporaneous usefulness of our entropy setting in market risk management. The degree of predictability is closely linked to both the type of entropy and the nature of the underlying signal.
\end{abstract}

Keywords: Decision analysis; Risk management; Financial Crisis; Entropy.

JEL No: G0; G1; C40

\footnotetext{
${ }^{*}$ Corresponding author. IÉSEG School of Management (LEM-CNRS), Lille Catholic University, 3, rue de la Digue, 59000 Lille, France. Phone: +33 (0) 320545 892. Email: n.gradojevic@ieseg.fr; Faculty of Technical Sciences, University of Novi Sad, Serbia; The Rimini Center for Economic Analysis, Italy.

${ }^{\dagger}$ Faculty of Economics and Engineering Management, Business Academy, Cvecarska 2, 21000 Novi Sad, Serbia.
} 


\section{Introduction}

The events that led to the financial crisis of 2008 were driven by a combination of behavioral, macroeconomic, regulatory and supervisory factors ${ }^{1}$. The interaction and build-up of such forces culminated in the months of September and October, 2008 that were marked by unprecedented price volatility. For instance, from October 6 to October 10, 2008, the S\&P 500 stock market index declined by $18.2 \%$, which represented its worst one-week loss since 1933. At the end of 2008, record losses were posted by all major stock market indices across the globe. In general, a stock market index reflects the current state of an economy, but it does not provide any advance information about a potential economic crisis. Furthermore, being an unbounded measure of the market's health, in crisis situations, a market index is unable to gauge the depth of the crisis.

This paper applies time-dependent, entropic measures of the financial market crisis risk from Gençay and Gradojevic (2010) and Pincus (1991) to assess the predictability of aggregate market fears in 2008. The primary objective is on tracking aggregate market expectations ahead and during the last quarter of 2008 when the crisis was highly pronounced. The findings for the crisis of 2008 confirm the ones for the crash of 1987: the entropy approach generated predictive signals prior to the extreme market movements. Another contribution of this work is in that it compares two types of underlying variables for the entropic risk measure - skewness premium and implied volatility - and shows that the volatility-based measure is inferior in terms of its predictive ability. We conclude that although volatility and entropy are related measures of market risk and uncertainty, entropy can be more useful in predictive modeling. Finally, we characterize the 2008 crisis from an entropic perspective and contrast it to the 1987 market crash. The results demonstrate that the (probability) distribution-free, approximate entropy approach is more appropriate to predict the sharp decline of the S\&P 500 stock market index in 2008.

In our analysis, we argue that the sentiment of a financial market can be summarized through the aggregation of the subjective expectations of its participants. If the expectations of market participants are highly dispersed and independent, systemic risk is relatively low and extreme price movements are less likely to occur. If however, market participants have highly dependent and less dispersed expectations, the aggregate market sentiment could drive prices to extraordinary levels. Our approach extracts aggregate market expectations from a past sequence of financial option prices and implied volatilities via time-dependent entropic methodologies. By utilizing these particular measures we concentrate on long-range, time-dependent, interactive instability and uncertainty in the stock

\footnotetext{
${ }^{1}$ In his seminal paper, Scholes (1996) summarizes potential causes of systemic risk.
} 
market (Martin et al., 2000). Moreover, the Tsallis (or a q-Gaussian) distribution that is estimated by maximizing the Tsallis entropy can capture the frequency of extreme events together with ordinary frequencies satisfactorily (Borland, 2002, 2004). In other words, the $q$-Gaussian distribution is convenient for management and estimation of risks in leptokurtic time series. In addition, we utilize a distribution-free approximate entropy that measures the complexity of the underlying variables in a time series setting.

In the recent years, the applications of entropy in finance and economics have increased considerably. Gell-Mann and Tsallis (2004), Tsallis (2009) and Tsallis (2011) nicely summarize those efforts. Scholarly contributions that link an entropic framework to the field of finance include Ishizaki and Inoue (2013), Namaki et al. (2013), and Bentes and Menezes (2012), while the paper by Gradojevic and Gençay (2011) provides an extensive review of major financial applications of the Tsallis entropy. The former papers concentrate on using entropy to understand turning points in foreign exchange rate time series (Ishizaki and Inoue, 2013), to study patterns in the non-extensitivity parameter $(q)$ in mature and emerging markets (Namaki et al., 2013), and to consider entropy in stock markets as an alternative to standard deviation (Bentes and Menezes, 2012). In other applications, Stutzer (2000) and Stutzer and Kitamura (2002) study option and asset pricing through an entropic methodology. Entropy-based measures of risk and rare-event probabilities can be found in Yang and Qiu (2005) and Kaynar and Ridder (2010). An important recent work concerns the application of directional entropy to traditional risk management tools such as the value-at-risk (Bowden, 2011).

The underlying variables that are used in constructing the time-varying entropy are extracted from a data set for put and call financial options written on the S\&P 500 stock market index. The main reason for such a choice is that option prices are discounted expectations of future outcomes of the underlying price. If we assume complete and frictionless markets, then there exists a risk neutral probability measure under which the call (or put) option price is given as the expected value of its discounted payoff at maturity. In a broader context, option prices represent the market's aggregate expectations and a set of put and call option price written on the S\&P 500 index (for the same maturity) can give an aggregate view of the outcome of the S\&P 500 index price on that maturity date. For instance, if the prices of deep out-of-the-money put options on S\&P 500 are relatively large compared to deep out-of-the-money call options, this may imply that the market expects a large downward movement in the price of the underlying. Essentially, such a situation suggests that there exists a high degree of market risk, but less uncertainty about future price movements.

In general, we deal with dispersed information and investigate how it is aggregated and 
reflected in the prices through the entropic measure. The main contribution of this paper is to adapt and extend the methodology from Gençay and Gradojevic (2010) to the 2008 financial crisis, which is based on measuring the distribution of a skewness premium as well as implied volatility ratio of financial option contracts through entropy in order to identify aggregate market fears ahead of a major market crash. Hence, the entropic approach brings a new perspective on proper management of financial risks. With this paper we hope to inspire novel research avenues and financial risk management techniques. This may lead to the use of entropy in market research (i.e., for making trading decisions and portfolio analysis) and integrate it into a trading platform, as it is the case with technical indicators nowadays.

In Section 2, we provide a brief overview of the Tsallis entropy and the motivation for its use. This is followed by a description of the approximate entropy in Section 3. In Section 4, we present the option data for the S\&P 500 index. The results are summarized in Section 5 , while the final section concludes the paper.

\section{Tsallis entropy}

To motivate the discussion, we consider the probability density function naturally derived from the variational principle related to the Tsallis entropy

$$
S_{q}(p(x))=k \frac{1-\int p(x)^{q} d x}{q-1}
$$

where $q$ is a measure of non-additivity such that $S_{q}(A \cup B)=S_{q}(A)+S_{q}(B)-(1-$ q) $S_{q}(A) S_{q}(B), k$ is a positive constant ( $k=1$ or $k=k_{B}$, where $k_{B}$ is the Boltzmann constant), and $p(x)$ is a probability density function.

$S_{q}$ in its discrete version can be written as

$$
S_{q}=\frac{1-\sum_{i=1}^{n} p_{i}^{q}}{q-1}
$$

where the number of states $i=1, \ldots, n, p_{i}$ is the probability of outcome $i$, and $n$ is the number of states.

One of the advantages of entropy in Equation (1) is that it yields power-law tails, which play an important role in finance. Natural constraints in the maximization of Equation (1) are

$$
\int p(x) d x=1
$$


corresponding to normalization, and

$$
\begin{gathered}
\int x \frac{[p(x)]^{q}}{\int[p(x)]^{q} d x} d x=\bar{\mu}_{q}, \\
\int\left(x-\bar{\mu}_{q}\right)^{q} \frac{[p(x)]^{q}}{\int[p(x)]^{q} d x} d x=\bar{\sigma}_{q}^{2},
\end{gathered}
$$

corresponding to the generalized mean and variance of a relevant quantity $x$, respectively.

Under the above constraints, one obtains

$$
p(x)=A_{q}\left[1+(q-1) B_{q}\left(x-\bar{\mu}_{q}\right)^{2}\right]^{\frac{1}{1-q}}, \quad q<3,
$$

where

$$
A_{q}=\frac{\Gamma\left[\frac{1}{q-1}\right]}{\Gamma\left[\frac{3-q}{1-q}\right]} \sqrt{\frac{q-1}{\pi}} B_{q}
$$

for the case that is of interest (when $q>1$ ), and $B_{q}=\left[(3-q) \bar{\sigma}_{q}^{2}\right]^{-1}$.

By defining the $q$-exponential function as

$$
e_{q}^{x}=[1+(1-q) x]^{\frac{1}{1-q}}
$$

one can rewrite Equation (6) as

$$
p(x)=A_{q} e_{q}^{-B_{q}\left(x-\bar{\mu}_{q}\right)^{2}},
$$

which is referred to as the $q$-Gaussian probability density function. Tsallis (1988) proposed this distribution to handle systems with long-range interactions, necessitating a nonextensive generalization of the ordinary Gibbs-Shannon entropy.

The main advantage of this distribution is, that in contrast to simple convolutions, which allow only for asymptotic behavior like $q=1$ (Gaussian) or $q>5 / 3$ (Lévy distribution) ones, the $q$-Gaussian distribution allows fat-tailed distributions associated with $1<q \leq 5 / 3$. 
As Gaussian distribution is unable to approximate fat tails (or extreme events) that are observed in many high-frequency empirical distributions in finance, we turn our attention to $q$-Gaussian probability distribution. Borland (2002) shows that $q$-Gaussian distribution provides a much better fit to the empirical distribution of high-frequency S\&P 500 index and Nasdaq index returns than the log-normal.

Through a moving window approach, the evolution of $S_{q}$ for the skewness premium and implied volatility ratio ${ }^{2}$ is calculated over time. ${ }^{3}$ The calculation of a time-dependent entropy is influenced by the following considerations (Thakor and Tong, 2004):

1. Number of states. With too few states, one may not be able to characterize the underlying market sentiment reliably, and with too many states, tracking fine changes becomes difficult. Without loss of generality, we set $n=10$.

2. Partitioning method. There are two different methods for partitioning the range of a time series: (a) fixed partitioning (equipartition is performed on all available data) and (b) adaptive partitioning (equipartition is performed on each moving-window of data, i.e., it changes over time). The adaptive partitioning approach can track transient changes better than the fixed partitioning and is more suitable for our application.

3. Estimation of $q$. The entropic index $q$ is the degree of long-memory in the data. GellMann and Tsallis (2004) estimate $q \approx 1.4$ for high-frequency financial data (returns and volumes) and stress that as the data frequency decreases, $q$ approaches unity. Larger $q$ values $(1<q \leq 2)$ emphasize highly volatile activities in the signal when a time-dependent entropy is plotted against time, i.e., the entropy is more sensitive to possible disturbances in the probability distribution function. In this paper, we find the optimal $q$ by using the maximum likelihood (ML) estimator, as explained in the Results section.

4. Sliding step $(\Delta)$ and moving window size $(K)$. The sliding step (the number of observations by which the moving window is shifted forward across time) and moving window size (the number of observations used in calculating the entropy) determine the time resolution of $S_{q}$. If the focus is on tracking the local changes, the sliding step is set to be very small (e.g., one observation: $\Delta=1$ ). Non-overlapping windows $(\Delta \geq K)$ are useful only when one is interested in monitoring the general trend of a time series. To get a reliable probability distribution function, $K$ should not be too small. We set $\Delta=1$ and $K=50$.

\footnotetext{
${ }^{2}$ These variables will be explained in the next section.

${ }^{3}$ See Gamero et al. (1997) or Tong et al. (2002) for more information.
} 


\section{Approximate entropy}

Approximate entropy is an index of complexity or uncertainty, for a given time series (Pincus, 1991). It is based on the likelihood that templates in a time series are similar to the next incremental comparisons. The templates are simply subsequences of the chosen time series. If a time series has a large approximate entropy, i.e., a larger diversity of patterns within a time series, then the time series is associated with a higher uncertainty or higher complexity. Essentially, approximate entropy is a distribution-independent statistic that is insensitive to outliers. It assigns a non-negative number to a time series with greater values corresponding to an increased randomness.

To calculate approximate entropy, the first step is to select a sequence $X=\left\{x_{1}, x_{2}, \ldots, x_{N}\right\}$ of length $N$, which will denote the time series. Then, for $m<N$, let

$$
u_{(m)}(i)=\left\{x_{i}, x_{i+1}, \ldots, x_{i+(m-1)}\right\}
$$

be an $m$-dimensional vector, starting at the $i$ th term in the sequence $\mathrm{X}$.

Let $u_{(m)}(i)$ and $u_{(m)}(j)$ be two vectors. The distance between the two vectors $u_{(m)}(i)$ and $u_{(m)}(j)$ of length $m$ is defined as

$$
d\left(u_{(m)}(i), u_{(m)}(j)\right)=\max \left\{\left|x_{i+k}-x_{j+k}\right|: 0 \leq k \leq m-1\right\} .
$$

Two vectors $u_{(m)}(i)$ and $u_{(m)}(j)$ are similar to each other if for some $r>0$,

$$
d\left(u_{(m)}(i), u_{(m)}(j)\right) \leq r
$$

where $r$ denotes the specified tolerance. Similar vectors are denoted by writing $u_{(m)}(j) \sim^{r} u_{(m)}(i)$.

Clearly, the tolerance level $r$ determines the maximum distance between the vectors $u_{(m)}(i)$ and $u_{(m)}(j)$. Now, in order to determine the number of similar vectors $u_{(m)}(j)$ for each of the $N-(m-1)$ vectors $u_{(m)}(i)$, the respective distances between the vectors must be measured.

Let $n_{(m)}(i)$ denote the number of vectors $u_{(m)}(j)$ similar to $u_{(m)}(i)$ for a fixed $i$ and $m$, where

$$
1 \leq n_{(m)}(i) \leq N-(m-1)
$$


The relative frequency of finding a vector $u_{(m)}(j)$ in the sequence $X$ that is similar to $u_{(m)}(i)$ within a tolerance level $r$ is defined as

$$
C_{(m)}\left(u_{(m)}(i) \mid X, r\right)=\frac{n_{(m)}(i)}{N-(m-1)} .
$$

Note that by Equation (13), it is clear that:

$$
\frac{1}{N-(m-1)} \leq C_{(m)}\left(u_{(m)}(i) \mid X, r\right) \leq 1 .
$$

Then, the average frequency of the logarithm of $C_{(m)}\left(u_{(m)}(i) \mid X, r\right)$ must be considered in the approximate entropy calculations.

The average frequency of the logarithm of Equation (14) is defined as

$$
\Phi_{(m)}(r)=\frac{1}{N-(m-1)} \sum_{i=1}^{N-(m-1)} \ln \left\{C_{(m)}\left(u_{(m)}(i) \mid X, r\right)\right\} .
$$

Now, using Equation (16), the approximate entropy algorithm may be defined.

The approximate entropy of length $m$ and sequence $X$ with a tolerance level $r$ is defined as

$$
A p E n_{(m)}(r):=\lim _{N \rightarrow \infty}\left(\Phi_{(m)}(r)-\Phi_{(m+1)}(r)\right) .
$$

Next, the approximate entropy for a sequence $X$ with a finite length $N$ can be determined using Equation (17).

Let $r$ denote the specified tolerance and $m$ denote the dimension of the vectors $u_{(m)}(i)$. For a finite sequence of length $N$, the approximate entropy is estimated by statistics using the following function:

$$
A p E n_{(m)}(r, N):=\Phi_{(m)}(r)-\Phi_{(m+1)}(r) .
$$

The parameters that are required to calculate the approximate entropy must be specified. Recall that $N$ is the length of the randomly chosen time series or sequence, $m$ denotes the dimension of the vectors $u_{(m)}(i)$ and $r$ is the specified tolerance level. In our analysis, $N$ denotes the length of the moving window which will be similar to $K$ for the Tsallis entropy, for the comparison purposes. Following Pincus (1991), we set $m=2$ and $r=0.15 \times \sigma$, where $\sigma$ represents the standard deviation of the time series. 


\section{Data}

The data are provided by DeltaNeutral and represent the daily S\&P 500 index European option prices, taken from the Chicago Board Options Exchange. Call and put options across different strike prices and maturities are considered for 2008. ${ }^{4}$ There are 173426 put options and 173403 call options in the sample, i.e., on average, about 690 put or call options are traded daily. Since it is one of the deepest and the most liquid option markets in the United States, the S\&P 500 index option market is sufficiently close to the theoretical setting of the Black-Scholes model. The data set also includes the implied volatility measure for each option given as a proprietary mean estimate by DeltaNeutral.

We calculate two underlying variables (predictors) for which the time-dependent Tsallis entropy is estimated: daily skewness premium and daily volatility ratio. The definition of skewness premium $(x)$ follows Gençay and Gradojevic (2010) and we use it for all available European options:

$$
x=\frac{P\left(S, T, K_{p}\right)}{C\left(S, T, K_{c}\right)}-1
$$

where $P$ is the put option premium, $C$ is the call option premium, $S$ is the price of the underlying (S\&P 500 Index), $T$ is maturity, $K_{c}$ and $K_{p}$ are strike prices for the deepest available out-of-the-money call and put options, respectively. Hence, we match put and call options of the same maturity and strike price, and use their prices to calculate the skewness premium.

For the same types of options, we also define a volatility measure that is supposed to capture market risk ahead of time. Volatility ratio $(v)$ is calculated as

$$
v=\frac{\sigma_{I V}\left(P\left(S, T, K_{p}\right)\right)}{\sigma_{I V}\left(C\left(S, T, K_{c}\right)\right)}
$$

where $\sigma_{I V}\left(P\left(S, T, K_{p}\right)\right)$ is the implied volatility of the deepest out-of-the-money put option and $\sigma_{I V}\left(C\left(S, T, K_{c}\right)\right)$ is the implied volatility of the deepest out-of-the-money call option, while the rest of notation is as previously defined. The motivation for this measure is to utilize a possibility that the volatility of put options (due to excessive purchases by riskaverse investors) may be larger relative to call options in advent of the financial crisis.

\footnotetext{
${ }^{4}$ Options are financial derivatives whose value (i.e., price) depends on the value of the underlying security. For example, a call (put) option based on a stock provides its buyer with a right to purchase (sell) a predetermined amount of stocks at a contracted price ("strike price") on or by a specific date ("maturity"). For this right the buyer of an option pays a price called the premium.
} 


\section{Results}

First, we estimate the long memory parameter $(\hat{q})$ based the first two quarters of 2008 (sample period before the crisis) by using the ML estimator as follows

$$
q=\underset{q}{\operatorname{argmax}} \prod_{i=1}^{T} p\left(x_{i} \mid q\right)=\underset{q}{\operatorname{argmax}} \sum_{i=1}^{T} \log p\left(x_{i} \mid q\right),
$$

where $\mathrm{T}$ is the sample size.

We find that $\hat{q}_{x}=1.62(0.04)$ and $\hat{q}_{v}=1.78(0.08) .{ }^{5}$ Using the optimal $q$, next, we investigate the evolution of the time-dependent Tsallis entropy based on the two underlying variables: $x$ and $v$.

Table 1 presents how the probabilities of the states are distributed before and during the crisis based on the skewness premium $(x)$. Before April, 2008, the probabilities were relatively evenly distributed across the states, although more weight can be found in states $s_{1}-s_{5}$. In the subsequent period, a convergence towards states $s_{1}-s_{3}$ can be observed and large probability values are located in states $s_{1}$ and $s_{2}$. For example, on July 1, 2008, the first two states carry a combined probability of 0.78 . The redistribution of probabilities is followed by a declining trend in the entropy values. This trend is strong until August 15, 2008, when the entropy dips to 0.606 and the market concerns are at their highest level since the beginning of 2008. The probabilities remained concentrated in states $s_{1}$ and $s_{2}$ until October 10, 2008, when, according to our framework, the financial crisis reaches its peak and the first state received $90 \%$ of the probabilities. The movements in the entropy along with the skewness premium can be tracked in Figure 1. As the financial crisis was evident in September of 2008 and the major stock market indices recorded largest losses in October of 2008, we consider the strong concentration of probabilities on August 15, 2008 as well as their subsequent stable distribution to be a confirmation of the predictive ability of our methodology.

Next, we will monitor the volatility ratio $(v)$ and the resulting entropy in Figure 2. In contrast to Figure 1, no clear predictive pattern in the entropy can be observed in the months prior to the crisis. The entropy oscillates between approximately 0.8 and 1.3 with several trend reversals. There exists a significant volatility outburst on October 17, 2008 that abruptly reduces the entropy from 1.316 to 0.065 . It remains low and stable afterwards, indicating the severity of the crisis in the last quarter of 2008. Given the absence of any

\footnotetext{
${ }^{5}$ The number in parentheses is the bootstrap standard error. We use one leave-out bootstrap with replacement for a window size of $K=50$ observations.
} 


\begin{tabular}{llllllllllll} 
Date & $s_{1}$ & $s_{2}$ & $s_{3}$ & $s_{4}$ & $s_{5}$ & $s_{6}$ & $s_{7}$ & $s_{8}$ & $s_{9}$ & $s_{10}$ & TE \\
\hline Apr 1, 2008 & 0.3 & 0.2 & 0.1 & 0.2 & 0.06 & 0.02 & 0.04 & 0.04 & 0.02 & 0.02 & 1.397 \\
May 1, 2008 & 0.24 & 0.22 & 0.28 & 0.2 & 0.02 & 0.02 & 0 & 0 & 0 & 0.02 & 1.236 \\
Jun 2, 2008 & 0.18 & 0.42 & 0.26 & 0.08 & 0.02 & 0.02 & 0 & 0 & 0 & 0.02 & 1.130 \\
Jul 1, 2008 & 0.56 & 0.22 & 0.08 & 0.06 & 0.02 & 0.02 & 0 & 0 & 0.02 & 0.02 & 1.005 \\
Aug 1, 2008 & 0.08 & 0.72 & 0.02 & 0.04 & 0.06 & 0.04 & 0 & 0 & 0.02 & 0.02 & 0.784 \\
Aug 15, 2008 & 0.02 & 0.74 & 0 & 0 & 0 & 0.02 & 0 & 0 & 0.02 & 0.02 & 0.606 \\
Sep 2, 2008 & 0.22 & 0.62 & 0.08 & 0 & 0 & 0 & 0.02 & 0.02 & 0.02 & 0.02 & 0.869 \\
Sep 15, 2008 & 0.24 & 0.46 & 0.24 & 0 & 0 & 0 & 0 & 0.02 & 0.02 & 0.02 & 1.014 \\
Oct 1, 2008 & 0.30 & 0.50 & 0.10 & 0.02 & 0 & 0.02 & 0 & 0.02 & 0.02 & 0.02 & 1.012 \\
Oct 8, 2008 & 0.48 & 0.32 & 0.08 & 0.04 & 0 & 0.02 & 0 & 0.02 & 0.02 & 0.02 & 1.033 \\
Oct 9, 2008 & 0.76 & 0.10 & 0.04 & 0.02 & 0.02 & 0.02 & 0.02 & 0 & 0 & 0.02 & 0.679 \\
Oct 10, 2008 & 0.90 & 0.06 & 0.02 & 0 & 0 & 0 & 0 & 0 & 0 & 0.02 & 0.299 \\
Oct 15, 2008 & 0.86 & 0.04 & 0.02 & 0 & 0.02 & 0 & 0 & 0 & 0.02 & 0.04 & 0.430 \\
Nov 3, 2008 & 0.80 & 0.04 & 0.04 & 0.04 & 0.02 & 0 & 0 & 0 & 0.02 & 0.04 & 0.595 \\
Dec 1, 2008 & 0.80 & 0.04 & 0.04 & 0.04 & 0.02 & 0 & 0 & 0 & 0.02 & 0.04 & 0.595 \\
& & & & & & & & & & & \\
\hline
\end{tabular}

Table 1: Distribution of Aggregate Market Expectations for Skewness PreMIUM

The time-dependent Tsallis entropy (TE) is calculated with a moving window of 50 days for the skewness premium $(x) . s_{1}, \ldots, s_{10}$ denote non-overlapping intervals (states). The lower boundary of $s_{1}$ is the minimum of the moving window. Accordingly, the upper boundary of $s_{10}$ is the maximum of the moving window. Aggregate expectation probabilities $\left(p_{i}\right)$ are calculated from the ratio between the number of observations in each interval and the total number of observations in the moving window. The maximum entropy (expectations heterogeneity) corresponds to equal probability of $10 \%$ for each state. The minimum entropy (expectations homogeneity) occurs when all observations concentrate in one particular state such that one state receives $100 \%$ of the probability. In this particular case above, one can observe how the distribution of expectations converges to states $s_{1}, \ldots, s_{3}$ in the months prior to the peak of the crisis (October 10, 2008, according to the entropic framework). The increased concentration leads to a reduction in the entropy. 


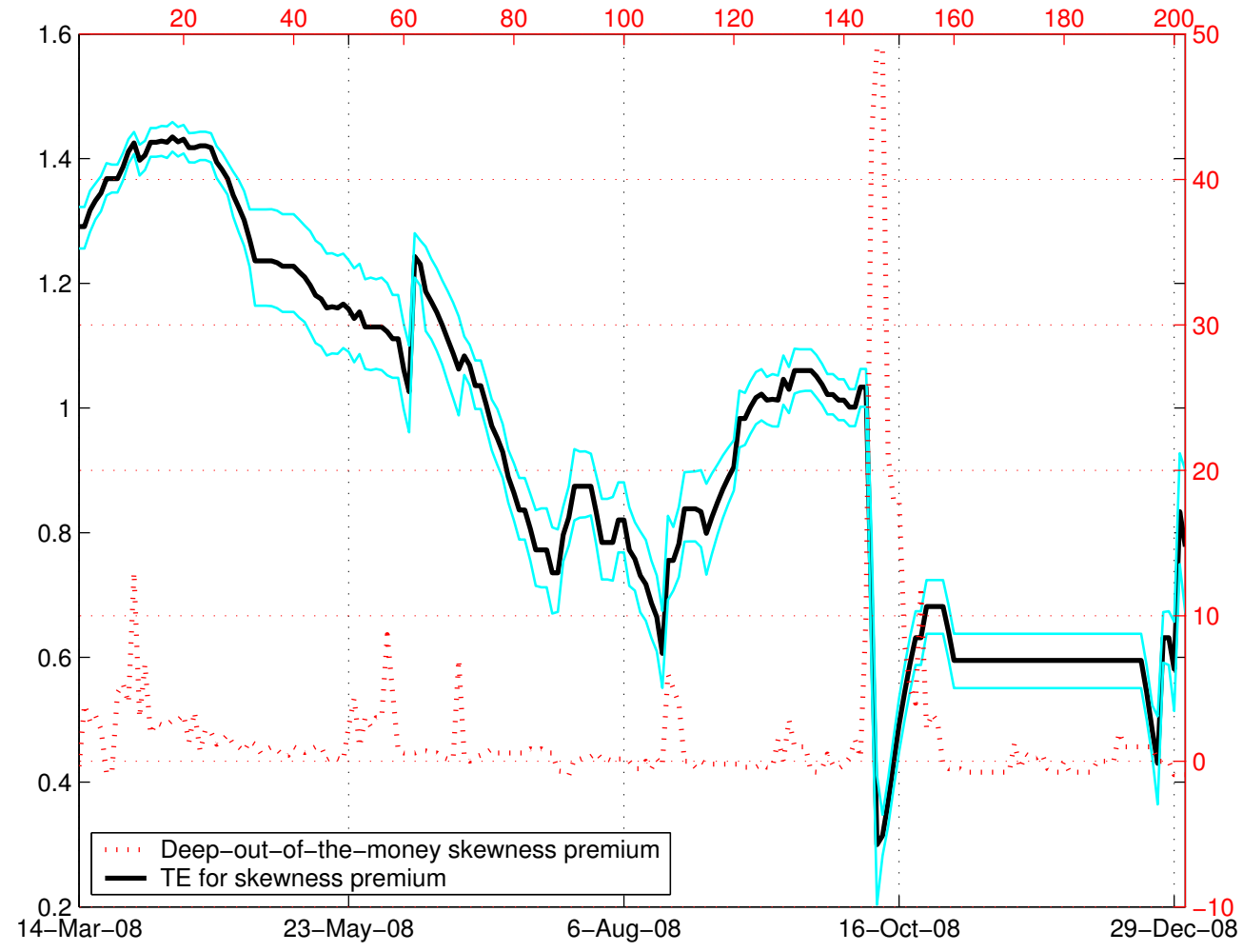

Figure 1: Skewness Premium and Dynamic Entropy

Tsallis entropy ( $T E$ ) values are plotted (solid line) along with the skewness premia for the deepest-out-of-the-money put and call options in 2008 (dotted line). The time-dependent, discrete entropy is calculated from the daily skewness premium based on the size of the moving window of $K=50$ days. The bootstrapped boundaries for the $95 \%$ confidence interval for the entropy are also displayed. The entropy exhibits a decreasing trend with a clear warning signal on August 15th, 2008, indicating strong aggregate market concerns during this period.

predictive signals, it can be concluded that the volatility-based entropy and the underlying volatility ratio are good contemporaneous indicators of the magnitude of financial crisis.

Finally, it would be useful to plot the skewness premium-based entropy next to the S\&P 500 stock market index. Figure 3 illustrates the predictive success of our framework. While the entropy exhibits a distinct downward trend from April, 2008 on, the S\&P 500 index does not take a substantial plunge until late May, 2008. Then, when the S\&P 500 index 


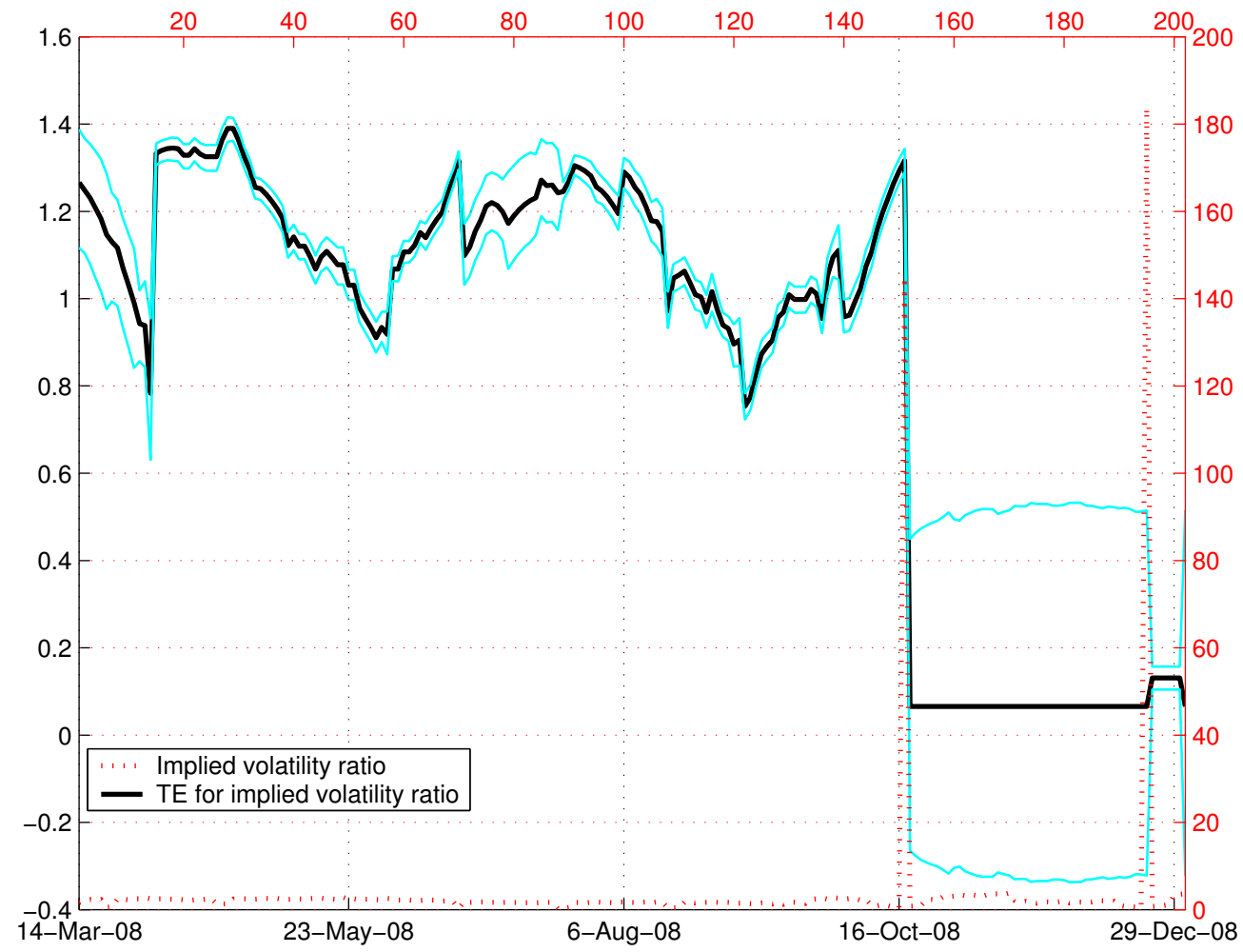

Figure 2: Implied Volatility Ratio and Dynamic Entropy

Tsallis entropy (TE) values are plotted (solid line) along with the implied volatility ratios of the deepest-out-of-themoney put to call options in 2008 (dotted line). The time-dependent, discrete entropy is calculated from the daily implied volatility measure based on the size of the moving window of $K=50$ days. The bootstrapped boundaries for the $95 \%$ confidence interval for the entropy are also displayed. The entropy does not exhibit a clear trend or a warning signal prior to the crisis, but drops substantially on October 20th, 2008.

was relatively stable in July and August, 2008, the entropy dipped and generated an early warning signal on August 15, 2008. The entropy trough that occurred on August 15, 2008 corresponds to a critical point with high degree of market risk and low uncertainty (i.e., low entropy) about the forthcoming crisis. Even though the entropy somewhat recovered in the following two months, the distribution of probabilities in Table 1 sends a clear message that the market risk was high and that the lack of aggregate expectations heterogeneity was still 


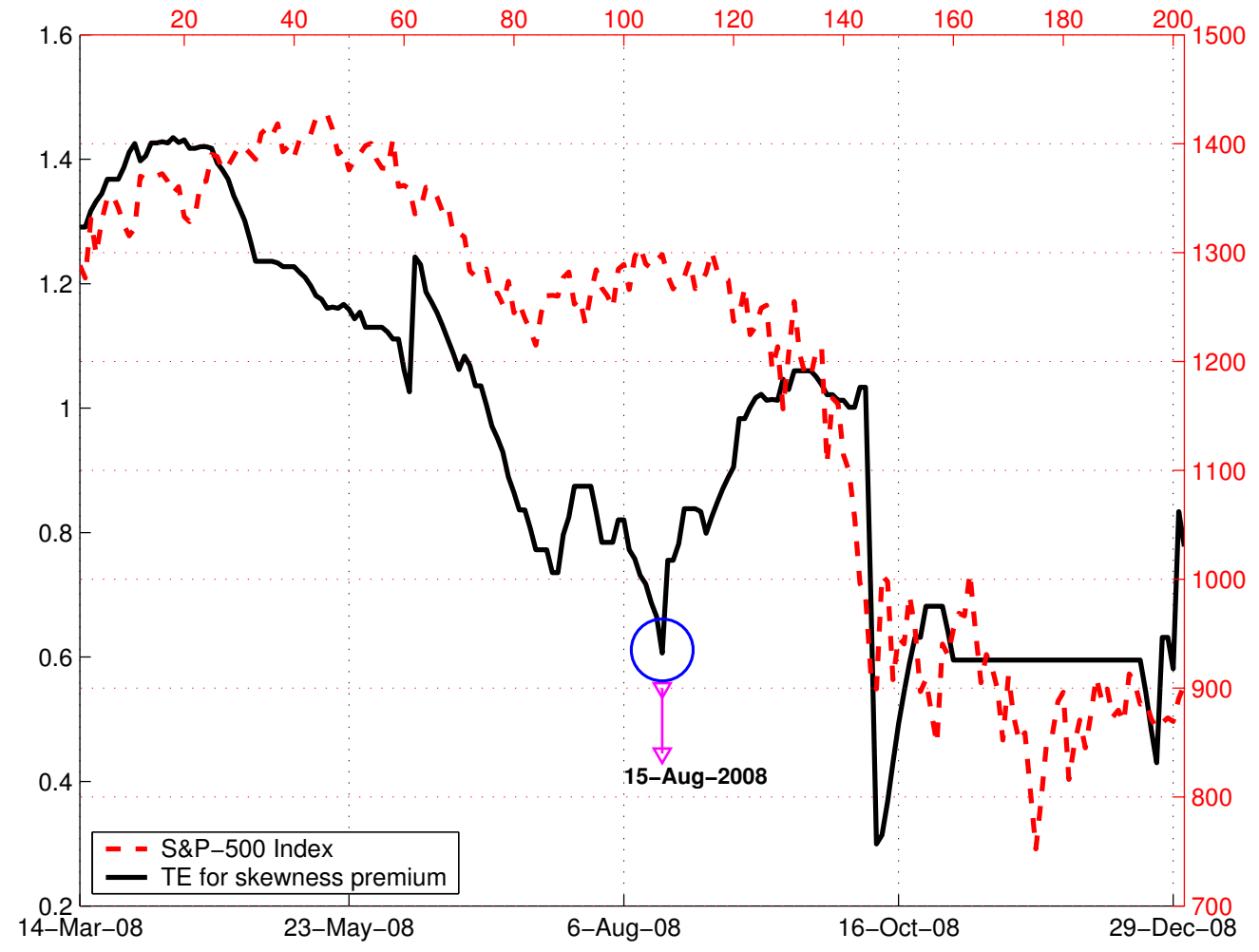

Figure 3: S\&P 500 InDEX AND DyNAMIC ENTROPY

Tsallis entropy (TE) values are plotted (solid line) along with the S\&P 500 Index levels in 2008 (dashed line). The time-dependent, discrete entropy is calculated from the daily skewness premium based on the size of the moving window of $K=50$ days. This figure illustrates the leading indicator property of the entropy with respect to the aggregate market movement as reflected in the S\&P 500 stock market index. The entropy bottoms out on August 15 th, 2008 which is indicated by the circle.

present over that period. ${ }^{6}$

Although the results presented above reveal major shifts in aggregate market expecta-

\footnotetext{
${ }^{6}$ It is important to stress that our findings are not sensitive to various reasonable choices for the size of the moving window $(K)$ and the number of states $(n)$, as well as the choice of non-extensitivity parameter $(q)$. For brevity, we do not provide the sensitivity analysis and the complete results can be obtained from the authors upon request.
} 
tions and systemic risk ahead of October, 2008, the fact that the Tsallis entropy recovers from its local minimum of August 15, 2008 may send a confusing signal regarding the predictability of the crisis (Figure 3). In Gençay and Gradojevic (2010), the early warning signal produced by the Tsallis entropy involved a strong decline about two months prior to the market crash, followed by low and stable entropy values until the crash date. Clearly, our framework shows that the Tsallis entropy is more accurate in predicting the one-day crash in 1987 than the peak of the crisis in 2008.

To investigate whether it is possible to obtain greater predictability, next, we employ the approximate entropy in the spirit of Pincus (1991) while the skewness premium remains the underlying variable. Figure 4 depicts the fluctuations in the skewness premia and the resulting approximate entropy. The feature that stands out in both panels is that they resemble Gençay and Gradojevic (2010) with respect to the plunge of entropy in the period before the crisis. Indeed, in Panel A of Figure 4, the entropy dips from about 0.75 to 0.3 on September 18, 2008 where it stays stable until the peak of the crisis. When we increase the vector (sequence) dimension to $m=10$ in Panel $\mathrm{B}$, the results become more striking with plenty of lead time until October, $2008 .^{7}$

\section{Conclusions}

This paper utilizes a time-dependent entropy approach in order to obtain an early indication of the 2008 financial crises. We extract the entropy from the daily skewness premia and implied volatility ratios for the put and call European options that were traded in 2008 . The results show that the entropic methodology performs well in tracking aggregate market expectations and generating early warning signals, thus, confirming the findings by Gençay and Gradojevic (2010) and Gradojevic and Gençay (2008).

The original contribution of the paper is threefold. First, we demonstrate that the volatility ratio and the volatility-based entropic risk measure lack a predictive ability and should be used mainly as contemporaneous indicators of the depth of the crisis. In this context, the skewness premium-based entropic risk measure is superior in forecasting movements of the S\&P 500 index. On the methodological side, we point to the necessity of studying and monitoring the time series plot of entropy values in tandem with the related discrete probability distribution. These two components complement each other and provide a complete picture of the likelihood of systemic risks. In the end, we directly compare the Tsallis entropy and the approximate entropy on a level playing field, and generate im-

\footnotetext{
${ }^{7}$ The results are not materially different when we vary the moving window size between $K=25$ and $K=75$.
} 
portant lessons in that respect. More specifically, we find that the Tsallis entropy is more appropriate in cases when the market movements are sudden and extreme such as in 1987. Large variability requires a non-extensive generalization of the ordinary Gibbs-Shannon entropy and $q$-Gaussianity. On the other hand, the crisis of 2008 had a relatively long build-up with frequent non-stationary signals for which the approximate entropy appears to be the dominant measure. In such situations, a distribution-free entropic approach for quantifying market expectation becomes more useful relative to an approach that abides by a specific distribution. 


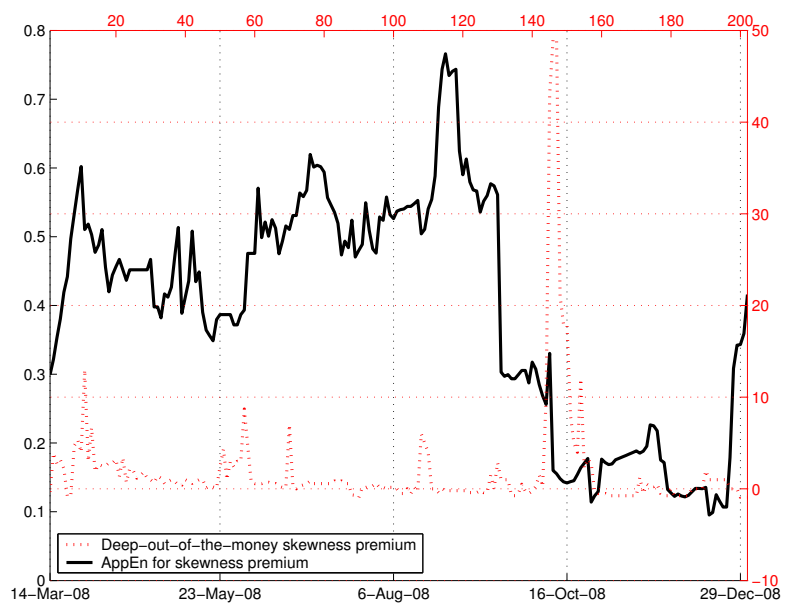

Panel A

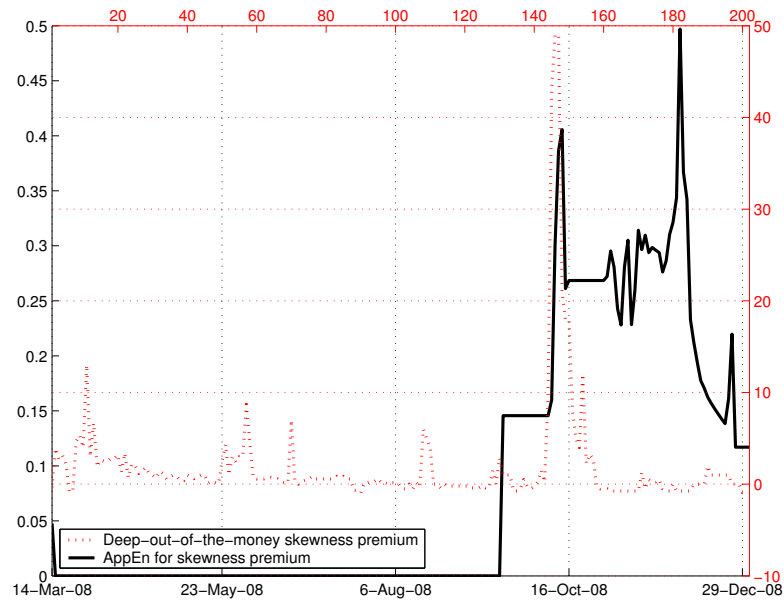

Panel B

\section{Figure 4: Skewness Premium and Approximate Entropy}

Notes: In Panel A approximate entropy $(A p p E n)$ values are plotted (solid line) along with the skewness premia for the deepest-out-of-the-money put and call options in 2008 (dashed line). The time-dependent, approximate entropy is calculated from the daily skewness premium based on the size of the moving window of $K=50$ days, vector dimension $m=2$ and $r=0.15 \times \sigma$, where $\sigma$ represents the standard deviation of the moving window. This figure shows a clear signal about a month before the major dip in the S\&P 500 index. Panel B is similar to Panel A, except that vector dimension $m=10$. This figure exhibits extremely low entropy values well ahead of October, 2008. 


\section{References}

Bentes, S. and Menezes, R. (2012). Entropy: A new measure of stock market volatility? Journal of Physics: Conference Series, 394(1).

Borland, L. (2002). A theory of non-gaussian option pricing. Quantitative Finance, 2, 415-431.

Borland, L. (2004). The pricing of stock options. In M. Gell-Mann and C. Tsallis, editors, Nonextensive Entropy: Interdisciplinary Applications, pages 305-320. Oxford University Press.

Bowden, R. J. (2011). Directional entropy and tail uncertainty, with applications to financial hazard. Quantitative Finance, 11(3), 437-446.

Gamero, L. G., Plastino, A., and Torres, M. E. (1997). Wavelet analysis and nonlinear dynamics in a nonextensive setting. Physica A, 246, 487-509.

Gell-Mann, M. and Tsallis, C. (2004). Nonextensive Entropy: Interdisciplinary Applications. Oxford University Press, Oxford.

Gençay, R. and Gradojevic, N. (2010). Crash of '87 - was it expected? Aggregate market fears and long range dependence. Journal of Empirical Finance, 17(2), 270-282.

Gradojevic, N. and Gençay, R. (2008). Overnight interest rates and aggregate market expectations. Economics Letters, 100(1), 27-30.

Gradojevic, N. and Gençay, R. (2011). Financial applications of nonextensive entropy. IEEE Signal Processing Magazine, 28(5), 116-121.

Ishizaki, R. and Inoue, M. (2013). Time-series analysis of foreign exchange rates using timedependent pattern entropy. Physica A: Statistical Mechanics and its Applications, 392(16), 33443350 .

Kaynar, B. and Ridder, A. (2010). The cross-entropy method with patching for rare-event simulation of large markov chains. European Journal of Operational Research, 207(3), 1380-1397.

Martin, M. T., Plastino, A. R., and Plastino, A. (2000). Tsallis-like information measures and the analysis of complex signals. Physica A, 275(1), 262-271.

Namaki, A., Lai, Z. K., Jafari, G., Raei, R., and Tehrani, R. (2013). Comparing emerging and mature markets during times of crises: A non-extensive statistical approach. Physica A: Statistical Mechanics and its Applications, 392(14), 3039-3044.

Pincus, S. M. (1991). Approximate entropy as a measure of system complexity. In Proceedings of the National Academy of Sciences, volume 88, pages 2297-2301.

Scholes, M. (1996). Global financial markets, derivative securities, and systemic risks. Journal of Risk and Uncertainty, 12, 271-286.

Stutzer, M. J. (2000). Simple entropic derivation of a generalized Black-Scholes option pricing model. Entropy, 2, 70-77. 
Stutzer, M. J. and Kitamura, Y. (2002). Connections between entropic and linear projections in asset pricing estimation. Journal of Econometrics, 107, 159-174.

Thakor, N. V. and Tong, S. (2004). Advances in quantitative electroencephalogram analysis methods. Annual Review of Biomedical Engineering, 6, 453-495.

Tong, S., Bezerianos, A., Paul, J., Zhu, Y., and Thakor, N. V. (2002). Nonextensive entropy measure of eeg following brain injury from cardiac arrest. Physica A, 305, 619-628.

Tsallis, C. (1988). Possible generalization of Boltzmann-Gibbs statistics. Journal of Statistical Physics, 52, 479-487.

Tsallis, C. (2009). Introduction to Nonextensive Statistical Mechanics: Approaching a Complex World. Springer, New York.

Tsallis, C. (2011). The nonadditive entropy Sq and its applications in physics and elsewhere: Some remarks. Entropy, 13, 1765-1804.

Yang, J. and Qiu, W. (2005). A measure of risk and a decision-making model based on expected utility and entropy. European Journal of Operational Research, 164(3), 792-799. 